\section{Producción hortícola \\ sustentable: una apuesta para \\ mejorar la calidad de vida de jóvenes en situación de riesgo}

\author{
María Belén Rosini \\ Docente investigadora e integrante \\ del Centro Regional de Estudio de \\ Cadenas Agroalimentarias (CRESCA) \\ de la Facultad de Agronomía. \\ Universidad Nacional del Centro \\ de la Provincia de Buenos Aires. \\ Participante de la Agencia de \\ desarrollo local del Municipio de \\ Olavarría (ADELO).
}

\author{
Ana María Castagnino \\ Docente investigadora e integrante \\ del Centro Regional de Estudio de \\ Cadenas Agroalimentarias (CRESCA) \\ de la Facultad de Agronomía. \\ Universidad Nacional del Centro \\ de la Provincia de Buenos Aires. \\ Anabella Sesto \\ Participante de la Agencia de \\ desarrollo local del Municipio de \\ Olavarría (ADELO).
}

Universidad y salud /

Intervenciones

\section{Resumen}

La actividad hortícola, entre sus múltiples posibilidades, representa una oportunidad de reinserción laboral para jóvenes en situación de riesgo (JSR) a través de implementación de proyectos productivos interinstitucionales con enfoque sistémico (PPIES). A fin de impulsar producciones hortícolas sustentables de calidad, como estrategia para la expansión del sector hortícola en Olavarría, la reinserción social de JSR y mejora de la calidad de vida de los habitantes, se generó el proyecto interinstitucional "Hacia la diferenciación de productos hortícolas con potencial regional: caso La Granja". Se efectuaron capacitaciones, implementación de protocolos y planillas de registro; se equipó un área de poscosecha y se acondicionó en fresco la producción lograda. La contribución del proyecto no sólo estuvo relacionada con mejores resultados productivos, en rendimiento y calidad, sino que además contribuyó a la mejora de factores psicológicos y emocionales observados en el cambio de actitud, lo cual aumentó las sensaciones positivas del grupo.

\author{
Palabras clave \\ - horticultura \\ - reinserción laboral \\ - capacitación \\ - jóvenes en situación de riesgo \\ - sustentabilidad
}

\section{Introducción}

La actividad hortícola, entre sus múltiples posibilidades, representa una oportunidad de reinserción laboral para jóvenes en situación de riesgo a través de la implementación de proyectos productivos con enfoque sistémico, para lo cual resulta imprescindible el logro de una adecuada articulación interinstitucional tendiente a una mejor calidad de vida de los jóvenes como consecuencia de su reinserción laboral y una mejor alimentación.

La horticultura se viene utilizando, en diferentes programas, como una actividad vehículo para el desarrollo personal de los individuos, el aprendizaje de habilidades básicas, el estímulo del funcionamiento físico y del aprendizaje, abordando habilidades de socialización, cooperación, trabajo en equipo y la incorporación de hábitos de vida saludables en un entorno al aire libre que lograrían el bienestar físico y mental.

El derecho a la alimentación es un derecho humano universal que insta a que las personas tengan acceso a una alimentación adecuada y a los recursos necesarios para tener en forma sostenible seguridad alimentaria, tal cual lo estipula la Organización de Naciones Unidas para la Alimentación y la Agricultura, FAO. De allí que resulte importante el aporte de las instituciones gubernamentales y la universidad pública para comprometerse activamente con los temas y problemas de la comunidad en la búsqueda de una mayor inclusión y una mejor calidad de vida. En tal sentido, según Balbín (2008) y Angarita Cañas (2002), las instituciones se constituyen en portadoras de responsabilidades en función del rol que asumen en la sociedad.

En tanto, Castro, Celma, Farías Toñánez y Gallagher Ovelar sostienen que.
"La educación y el trabajo son derechos humanos y además constituyen un requisito fundamental para el acceso al empleo de calidad, circunstancia ésta que se acrecienta en un contexto de mundialización, regionalización, tecnologización y de advenimiento de la denominada sociedad del conocimiento. En tal sentido, la educación, y en particular la formación profesional, debe ser mirada, necesariamente, como una dimensión que contribuye a la concreción del trabajo decente y del pleno empleo" (2010:24).

Uno de los ejes fundamentales del desarrollo socioeconómico y cultural de un país está conformado por la calidad de la educación, la competitividad del sector productivo y las políticas activas de empleo, según Castro y otros (2010).

Los partidos que, como Olavarría, integran la zona centro de la provincia de Buenos Aires, presentan un gran potencial para las alternativas productivas intensivas como las hortícolas, las que requieren para su posicionamiento estrategias concretas de diferenciación, para lo cual es necesario efectuar capacitaciones tendientes a la generación de jóvenes emprendedores. Al respecto, los municipios, las universidades, las entidades educativas y productivas tienen la posibilidad de contribuir a la formación de nuevos emprendedores a partir de la creación de vínculos interinstitucionales que, además, contribuyan a brindar oportunidades concretas a jóvenes en situación de riesgo. Los mundos de la educación y del trabajo son realidades diferenciadas con sus propias lógicas de interacción y sus códigos de comunicación, según Castro y otros (2010). El mundo de la 


\section{6}

la actividad hortícola representa

una oportunidad de reinserción laboral para jóvenes en situación de riesgo

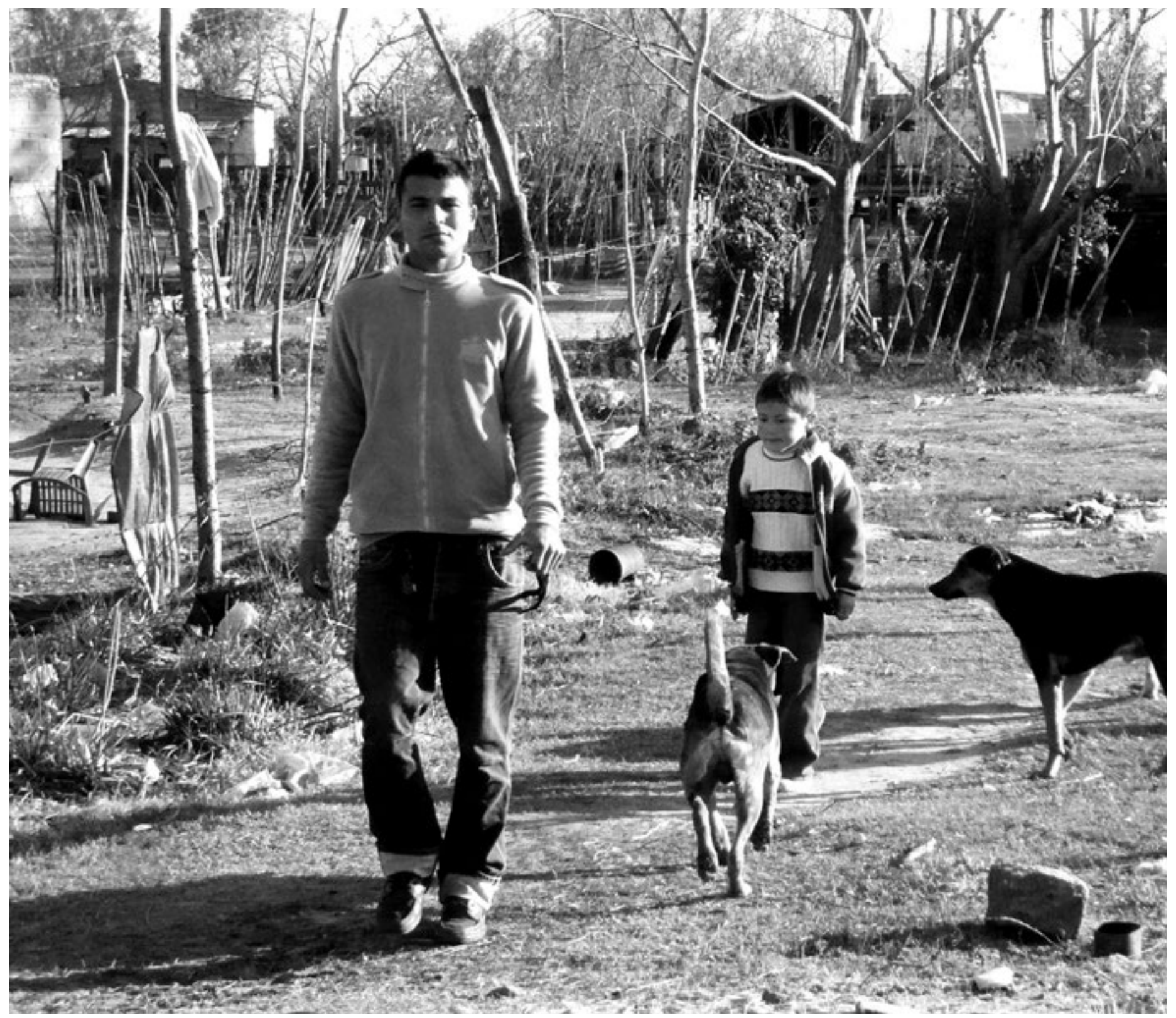


educación debería ser el de la equidad, la homogeneidad, la universalidad y la calidad (Riquelme y Herger, 2006), mientras que el de la producción es un mundo donde predomina la diversidad y en el que es necesario esforzarse por armonizar los conceptos de sustentabilidad, productividad y competitividad. Ambos mundos no son compartimentos estanco con límites definitivos y excluyentes sino que se complementan y se enlazan entre sí y con otros sistemas para dar cuerpo a un conjunto social organizado. El esfuerzo por comprender cada uno de ellos permitiría su desarrollo de forma tal que favorezca el despliegue de las potencialidades de las personas (Castro y otros, 2010), para lo cual resulta imprescindible la articulación interinstitucional, de manera de propiciar el logro de objetivos previamente consensuados. Y la interacción entre educación y trabajo permite la incorporación competencias laborales (Castro y otros, 2010:30).

La capacitación profesional, según Catalano (2004), puede ser entendida como un proceso de desarrollo individual tendiente a adquirir y perfeccionar capacidades que permitan ejercer de la mejor forma un oficio, una profesión o un trabajo. Implica la adopción de habilidades y destrezas, que le permitan, por ejemplo, llevar adelante un proyecto productivo.

La articulación interinstitucional orientada a la implementación de servicios de educación facilita una base real al concepto de formación continua a lo largo de toda la vida, según Castro y otros (2010:35).

El mercado de trabajo, compuesto por la población

económicamente activa, que no es propietaria de los medios de producción, está integrado inclusive por jóvenes que requieren habilidades que les posibiliten una adecuada inserción en el mundo del trabajo, tal como lo indica Sladogna (2000), evitando los riesgos a los que de otro modo se verían sometidos.

La Universidad del Centro de la Provincia de Buenos Aires (UNCPBA) ha desarrollado un programa de extensión con el objetivo de vincular la docencia e investigación con la comunidad y lograr una articulación que aporte soluciones a las problemáticas y necesidades de la sociedad mediante la intervención. Desde las políticas de la UNCPBA se entiende a la extensión como un proceso de conocimiento nuevo, que vincula críticamente el saber científico con el saber popular. Asimismo, es una función que admite orientar líneas de investigación y planes de enseñanza generando compromiso universitario con la sociedad que apunte a la resolución de sus problemas. De esta manera, mediante los diferentes proyectos se consigue materializar, en un conjunto variado de actividades y acciones planificadas, la utilidad social del conocimiento y la formación solidaria, contribuyendo al mejoramiento tanto de la sociedad como del individuo. De ellos surgen propuestas de intervención, servicios, asistencia técnica, producción y/o transferencia de conocimientos y resultados de investigación que atienden a problemáticas definidas a través de estrategias explícitas en contextos delimitados.

En tal sentido, se generó un proyecto de producción hortícola sustentable ecoeducativo, como ejemplo regional, orientado a impulsar la producción sustentable y diversificada como estrategia para la expansión del sector en la zona, la mejora de la calidad de vida de los habitantes y la reinserción social de jóvenes en situación de riesgo. Entre los objetivos específicos que se plantearon, figuran: - Capacitar a jóvenes en situación de riesgo en los distintos sistemas de producción hortícola.

- Facilitar tecnologías sustentables de procesos y no de insumos, tales como: la asociación de cultivos, la inclusión de aromáticas, el manejo integrado de plagas, entre otros.

- Evaluar los cultivos de mayor calidad obtenidos y, sobre ellos, trabajar en la incorporación de packaging especiales como estrategia para la diferenciación y valorización de las hortalizas producidas.

- Brindar conocimientos que permitan el autosustento y una salida laboral a jóvenes en situación de riesgo.

Con estos propósitos se busca principalmente capacitar a los beneficiarios para promover en ellos nuevas habilidades en actividades demandantes de mano de obra, generar actitudes para el trabajo, hábitos y salida laboral, o al menos los conocimientos que permitan el autosustento. A su vez, el proyecto apunta a generar recursos necesarios para enriquecer la dieta y satisfacer la demanda de alimentos para sustento de la familia de los beneficiarios. Es muy gratificante para los jóvenes llevar un producto cuidado por ellos a su casa, como también lo es el incentivo ecónomico originado por las ventas de excedentes. Para este proyecto se utilizó a la actividad hortícola como instrumento educativo y socializador, ya que ésta, entre sus múltiples oportunidades, representa una alternativa de reinserción laboral para jóvenes en situación de riesgo. Los emprendimientos productivos constituyen espacios económicos interesantes en aquellos casos en los que el acceso al empleo y la generación de ingresos se ven limitados.

La institución elegida para desarrollar el proyecto fue el Centro de Día N 3 "La Granja", perteneciente al Municipio de Olavarría. Desde hace dos décadas, el Centro realiza distintas actividades de capacitación y de inserción laboral para jóvenes en situación de riesgo. Además, se los contiene y acompaña para superar problemas familiares, de deserción escolar y de riesgo social. Los jóvenes en la institución terminan sus estudios primarios y deben egresar con la posibilidad de ser insertados en el medio laboral o continuar con sus estudios por medio de tutorías. El número de alumnos que asiste es variable. Durante el período del presente proyecto dicho número fue de 30 jóvenes.

El predio de la institución cuenta con dos hectáreas en las cuales se encuentran la zona de aulas, talleres, depósito de herramientas e insumos, comedor, zona de recreación con pileta, canchas de fútbol y voley. La zona de producción está comprendida por áreas destinadas a pequeños animales de granja, huerta y frutales, y un área de acondicionamiento y venta. Esta última fue incorporada como uno de los objetivos planteados para este proyecto. Se 
cuenta con un responsable de las tareas de producción y dos docentes a cargo del grupo.

Las actividades productivas que llevan a cabo son la cría de pequeños animales, como gallinas ponedoras, conejos, ovejas, y la huerta, que se realiza a campo abierto y bajo invernadero. Gracias al presente proyecto se mejoraron las técnicas de producción de hortalizas. La duración del proyecto fue de un año, comprendido en el período de noviembre de 2012 a noviembre de 2013. Se trabajó desde la capacitación, producción y diferenciación de productos hortícolas, con base en tecnologías de procesos y no de insumos, o sea que todos sus componentes se encontraron en equilibrio con el medio ambiente, tanto para la preparación del suelo como para el cultivo y el procesado.

Siempre se hizo hincapié en la contención de los jóvenes, por lo que se trabajó el proyecto desde la producción hortícola sustentable ecoeducativa, desde la sensibilización en temáticas relacionadas con ambiente, nutrición y salud. Los jóvenes fueron capacitados en el diseño, ejecución y mantenimiento de una huerta; se les brindaron técnicas de poscosecha para mantener las características del producto recién cosechado y se los introdujo en el empleo de técnicas de packaging tendientes a la valorización de los productos, como, por ejemplo, en empleo de calcomanías identificatorias para el armado de atados de puerro, cebolla de verdeo y cultivos de hoja como acelga, lechuga, entre otros.

\section{Metodología de la intervención}

Docentes de la Universidad realizaron visitas semanales durante todo el período del presente proyecto. En las primeras semanas, se efectuó un diagnóstico para determinar en cuáles de los cultivos que se venían llevando a cabo era más conveniente enfocar este proyecto y qué nuevos cultivos resultaba conveniente introducir. Una vez concluida la etapa de diagnóstico, desde la Universidad se entregaron insumos para las etapas de producción y de procesado. En el primer caso se aportaron semillas y plantines de aromáticas para la realización de las siembras, almácigos y plantaciones nuevas. En el caso de la etapa de procesado, se aportaron distintos elementos en la generación de un espacio específico o sector de procesado poscosecha para llevar a cabo las tareas de acondicionamiento de todos lo producido. Además se aportaron materiales para crear un sistema de semiforzado para producción a campo cuando las condiciones climáticas no lo permiten, como es el empleo de microtúneles.

Se planificaron las etapas de inicio de los cultivos, ya sea almácigo o siembra directa, o por plantación en el caso de las aromáticas. Como complemento se diseñaron e incorporaron al sistema productivo planillas de registro de datos de cultivo, es decir, todas las tareas que se realizan en el campo sobre cada cultivo, y otras planillas de registro de cosecha.

Se efectuaron capacitaciones a alumnos y docentes a cargo del sector productivo sobre diferentes técnicas de cultivo, requerimientos de cada especie a cultivar, especies aromáticas asociadas a la producción hortícola, control integrado de plagas, entre otras. También se capacitó en conceptos básicos para un adecuado manejo de los invernaderos y microtúneles. En las capacitaciones se hizo hincapié en el aprendizaje mediante la práctica a campo.

Se incorporaron técnicas de buenas prácticas de cosecha y poscosecha. Se realizaron reuniones de trabajo entre todas las instituciones intervinientes, con el objetivo de proponer los posibles envases, logo y descripción del producto. Las tareas se difundieron en medios locales y regionales y con ello se incentivó a la población a acercarse a la institución y a consumir hortalizas de producción local. En síntesis, se adecuaron las prácticas didácticas y los criterios de evaluación al perfil de este proyecto en particular.

\section{Condición previa a la práctica de extensión}

Los productos obtenidos en la huerta del Centro de Día "La Granja" tienen variados destinos. Principalmente son utilizados en la elaboración de los alimentos consumidos en el comedor de la institución y, como complemento, una vez por semana los alumnos cosechan y se llevan hortalizas a su casa. Los excedentes son comercializados a otros Centros de Día, Jardines Maternales, y a 
particulares que se acercan a la Granja con el deseo de obtener un producto fresco y de calidad.

Al ser tan variadas las especies que se producen, se emplean diferentes formas de producción según las especies producidas en cada época del año.

Las hortalizas producidas a lo largo del año son: lechuga, acelga, espinaca, achicoria, rúcula, perejil, habas, arvejas, coliflor, remolacha, zanahoria, papa, tomate, pimiento, berenjena, zapallo anco, de tronco, cebolla, puerro y ajo. El objetivo de la institución es que, de producirse excedentes de plantines o semillas, los jóvenes se los lleven a sus hogares a fin de replicar en ellos las técnicas aprendidas en la institución.

Con anterioridad al inicio del presente proyecto, el Centro de Día "La Granja" había recibido del Programa Huertas Bonaerenses, perteneciente al Ministerio de Asuntos Agrarios de la Provincia de Buenos Aires, herramientas de mano y semillas, animales de granja y plantas frutales del Programa PROHUERTA del Instituto Nacional de Tecnología Agropecuaria, INTA.

Los docentes de la institución, venían recibiendo algunas capacitaciones en temáticas afines al presente proyecto, como por ejemplo nociones básicas de huerta familiar, buenas prácticas en la producción hortícola, poda de frutales, elaboración de dulces y conservas, entre otras, las que actuaron de motivadoras hacia la integración interinstitucional en el marco del presente proyecto. La institución dispone de dos invernáculos tipo túnel ${ }^{1}$, donde realiza la mayor parte de las producciones hortícolas, ya que las particulares condiciones climáticas de la ciudad no permiten llevar a cabo con éxito las mismas, debido a las excesivas heladas del invierno y el intenso calor en verano.

Las tareas de siembra, carpida y cosecha se realizaban de manera manual; el acondicionamiento del suelo se realizaba de manera semimecanizada con motocultivador y el riego dentro de los invernáculos era por goteo y en la zona a campo de manera manual.

\section{Situación que se deseó modificar}

Con la implementación del proyecto se buscó:

- mejorar las técnicas productivas y la calidad de las producciones logradas en la institución y diferenciar a nivel local las especies hortícolas en las que se pueda obtener un producto de calidad, como así también contribuir a la optimización y diversificación de la dieta de los alumnos participantes del proyecto;

- generar un efecto multiplicador para otras instituciones educativas y microemprendedores hortícolas que, con los mismos objetivos, contribuyan a la expansión de las hortalizas a nivel regional; - generar mayores excedentes con la implementación de técnicas de manejo adecuadas, que le permita a la institución, comercializarlos a través de diferentes canales.

1) Los túneles son de $23 \mathrm{~m} \times 5 \mathrm{~m}$

y $40 \mathrm{~m} \times 7 \mathrm{~m}$.

\section{Vinculación con los actores} sociales con los que se trabajó

Con el objetivo de aunar esfuerzos, se realizó este proyecto interinstitucional de manera de que cada sector contribuya, cumpliendo el compromiso que tienen los organismos públicos hacia el desarrollo social y económico de los individuos. Las entidades coparticipantes de la Universidad fueron:

- Centro de Día № 3 "La Granja" perteneciente al área de Desarrollo Local del Municipio de Olavarría.

- Agencia de Desarrollo Local Olavarría (ADELO), perteneciente al Municipio de Olavarría. La Agencia aportó cuatro técnicos, un Ingeniero Agrónomo, una Licenciada en Alimentos, un diseñador (quien realizó el logo de las etiquetas y cintas para la venta) y personal administrativo (que se encargó de llevar adelante la carga de datos de las planillas de producción y venta).

\section{Cambios efectivamente producidos durante el proceso de la intervención}

Entre los resultados logrados se destacaron: la elaboración e implementación de protocolos de producción de las especies que se producen con mayor calidad y la realización de planillas de registro de insumos, siembra, transplante, manejo de plagas, cosecha y procesado. Se acondicionó y equipó un sector para poscosecha y acondicionamiento en fresco; colocando una mesa y una balanza. Además, se les entregó a los docentes de la granja, doce metros de caño ángulo y electrodos para que en el área de soldadura se fabrique un armazón exhibidor para las hortalizas no perecederas o para especies que requieran algún tipo de maduración. Se elaboró un listado de precios para poder homogeneizar los valores de venta, de modo que los valores cobrados sean acordes a los precios de mercado.

Se incorporaron aromáticas al sistema de producción y se diseñó y ejecutó una nueva plantación de dichas especies. Dicha plantación se efectuó con el aporte de plantines de lavanda, salvia, orégano y tomillo, adquiridos por la UNCPBA en el marco del proyecto.

Previa capacitación, se efectuaron prácticas de viverismo, siembra, cosecha y valorización de diversos cultivos hortícolas y de aromáticas medicinales, siguiendo las indicaciones propuestas en los protocolos y llevando los registros correspondientes. El impacto social que se destacó es la apropiación del proceso de producción y por consiguiente del proyecto, contribuyendo a mejorar las habilidades y la calidad de vida de los jóvenes involucrados. Los conocimientos adquiridos por los adolescentes y adultos referentes del Centro de Día continúan siendo aplicados, acompañando los distintos procesos productivos iniciados. Los logros en calidad y cantidad de lo producido actúan como un importante factor de autoestima para los adolescentes, como la concreción y educación que con esfuerzo, responsabilidad y con conocimientos específicos pueden mejorar y cumplir los objetivos propuestos en tiempo y forma. Para ellos fue importante observar que 
el proyecto tuvo continuidad durante el año de trabajo y los objetivos que se fueron proponiendo (tanto productivos como compra de herramientas, etc.) se fueron concretando en los plazos estipulados. Este proyecto integral pudo ser aplicado en todas sus dimensiones, por las distintas propuestas educativas que funcionan en el Centro de Día, ya que también se trabajó coordinadamente con la escuela primaria y secundaria que funcionan en la misma institución. Económicamente, desde su momento fundacional, lo producido en el Centro de Día, como ya se ha expresado, se consume internamente en el comedor escolar de la institución, los viernes se llevan los alumnos a sus casas y los excedentes se venden. La mejora en la producción con la nueva planificación propuesta, ha evidenciado que en todo momento, la Granja cuenta con hortalizas para consumo interno y venta de excedentes. Este aspecto ha tenido un impacto relevante, tanto para la economía interna del Centro de Día, que es destinada principalmente para actividades recreativas de los adolescentes como viajes, campamentos, etc., como a nivel personal de cada uno de los adolescentes donde, la venta de hortalizas en forma particular ha representado para ellos un aumento monetario que se suma al sistema de Becas que ya cuenta la Granja. Esta suma monetaria adicional ha impactado positivamente en la economía familiar de cada uno de los adolescentes.

Hubo efectos que superaron las expectativas, vinculados al compromiso con el proyecto, que asumieron los encargados de la huerta y los alumnos, ya que todos ellos han colaborado en las distintas actividades que se realizaron, como así también se han responsabilizado de mantener en el tiempo las consignas de buenas prácticas agrícolas que fueron incluidas en la producción. Todas las actividades propuestas fueron incorporadas por la Institución. Inclusive, la realización de microtúneles como complemento de las actividades originalmente previstas en el proyecto inicial para hacer más eficiente la producción de invierno y de verano al aire libre. Se explicó la técnica, se adquirieron los materiales, para que la institución pudiera armarlos.

La comercialización mejoró durante el transcurso del proyecto, si bien sigue siendo un punto que convendría fortalecer, dado que los docentes se encuentran abocados principalmente a la producción y el acompañamiento de los adolescentes. La mayor demanda se debe a que se ha fomentado la venta a particulares por medio de la difusión boca en boca, redes sociales y la comunicación en los medios.

Se incorporó la venta de hortalizas en las exposiciones y encuentros de diferentes centro que se hacen dentro del Centro de Día "La Granja".

La meta que se propuso con los integrantes de este proyecto fue aumentar los puntos de venta fijos en verdulerías, donde se puedan llevar los excedentes de la producción, sin que los encargados de la huerta deban preocuparse en plena producción por este punto. Sería interesante que la institución rediseñe la dieta del comedor de la institución, donde almuerzan diariamente, aumentando el porcentaje de hortalizas previsto.

\section{Conclusión}

El logro de una verdadera construcción interinstitucional, mediante el aporte de docentes, directivos, personal técnico y administrativo, permitió lograr la capacitación de los jóvenes en situación de riesgo del Centro de Día "La Granja", de manera que los mismos, no sólo adquirieran destrezas y habilidades, sino también el deseo de lograr una mejora continua de éstas y su aplicación como forma de vida. Los adolescentes lograron capacitarse en diversas producciones hortícolas y aromáticas, en invernadero y a campo, adoptando la modalidad de registro de todas las actividades y la adopción del concepto de buenas prácticas agrícolas y la importancia de valorización de los productos logrados. En tanto, los docentes tuvieron la oportunidad de desarrollar su profesionalidad y capacidades didácticas, incentivando el espíritu creativo e innovador de los alumnos. El personal directivo, técnico y administrativo desarrollaron capacidades para gestionar las acciones de vinculación con el medio. La vinculación con el contexto socio productivo y cultural, lograda en el marco de este proyecto, también se vio favorecida por la positiva valoración por parte de la comunidad respecto de los productos comercializados debidamente identificados.

La evaluación institucional y seguimiento de las actividades, desde la UNCPBA y ADELO y su valoración de los resultados logrados y de los efectos de las acciones realizadas, y su difusión, fueron el complemento necesario para el cierre de un ciclo positivo, que se reinicia anualmente en la institución Centro de Día "La Granja".

\section{Bibliografía}

Angarita Cañas, P. (2002). "La seguridad ciudadana: ¿Nuevo reto en la defensa de los derechos humanos?”. Revista lidh. v. fasc. pp. 117-166. San José de Costa Rica. Ayala, J. (2005). Competencias básicas en gestión. 1ra. ed. Buenos Aires: Banco Interamericano de Desarrollo.

Balbín, J. (2008). Metodología para la inclusión del enfoque de derechos en el desarrollo vía los planes. Medellín: Instituto Popular de Capacitación. Disponible en: http://www.ipc.org.co/images/stories/EnfoqueDerechos.pdf

Castro, J., Celma, L., Farías Toñánez, A. y Gallagher Ovelar, W. (2010). Capacidades del sistema público de formación profesional para incluir jóvenes en condiciones de vulnerabilidad. Disponible en: http://www.activamente.com.py/ sites/default/files/sdp_01_2010_1_informe_de_estudio_de_relevamiento.pdf (consultado el 10/04/2014).

Catalano, A. (2004). Diseño curricular basado en normas de competencia laboral. Buenos Aires: BID/FOMIN; OIT/Cinterfor.

FAO (s/f). Derecho a la Alimentación. Disponible en: http://www.rlc.fao.org/es/ iniciativa/la-iniciativa/derecho-alimentacion/

Riquelme, H. y Herger, N. (2006). "Escenarios de educación y formación para el trabajo: ¿Mercado de ilusiones de corto plazo o alternativas socio-educativas a la exclusión? Ponencia presentada en Meeting of the Latin American Studies Association. San Juan, Puerto Rico.

Sladogna, M. (2000). La recentralización del diseño curricular. El perfil profesional y la definición de competencias profesionales. Buenos Aires: Instituto nacional de educación tecnológica. Dirección nacional de educación técnico profesional. 